\title{
Functional impact of a recently identified quantitative trait locus for hippocampal volume with genome-wide support
}

\author{
S Erk ${ }^{1,2,12}$, A Meyer-Lindenberg ${ }^{3,12}$, P Schmierer ${ }^{1,2}$, O Grimm ${ }^{3}$, H Tost $^{3}$, T Mühleisen ${ }^{4}$, M Mattheisen $^{5}$, N Seiferth ${ }^{1}$, C Cichon $^{6}$, \\ M Rietschel ${ }^{7}$, MM Nöthen ${ }^{8,9}$, A Heinz $^{13}$ and $\mathrm{H}_{\text {Walter }}{ }^{1,2,10,11,13}$
}

In a large brain-imaging study, a multinational consortium has recently identified a common genetic variation in rs7294919 being associated with hippocampal volume. Here, we explored whether this quantitative trait locus also affects hippocampal function using a previously established reliable neuroimaging paradigm. We observed a significant effect of rs7294919 variation in the right hippocampus showing that hippocampal activation increased with the number of risk alleles. Furthermore, the risk allele was associated with decreased performance in a verbal learning and memory task. By showing that this single-nucleotide polymorphism also relates to behavioral difference and underlying brain activation in memory, our findings support the idea that rs7294919 may affect the individual capacity to resist disease in terms of diminishing or boosting hippocampal resources.

Translational Psychiatry (2013) 3, e287; doi:10.1038/tp.2013.57; published online 23 July 2013

\section{Introduction}

Identifying genetic variants that increase the risk for a heritable disease may not only reveal the underlying biological mechanisms but might also provide new treatment targets. In one of the largest brain-imaging studies ever, a multinational consortium (ENIGMA, enhanced neuroimaging genetics through meta analysis) recently identified a common genetic variation in rs7294919 located on chromosome 12q24 being associated with hippocampal volume $(n=21,151 ; P=6.7$ $\left.\times 10^{-16}\right):{ }^{1,2}$ an increased number of rs7294919- $T$ risk alleles was associated with decreased hippocampal volume. Hippocampal volume is highly heritable and implicated in many (neuro)psychiatric disorders, such as schizophrenia, bipolar disorder, major depression and Alzheimer's disease. The hippocampus is a key brain structure for memory formation and stress regulation, brain functions that are strongly implicated in the above mentioned disorders. The identified quantitative trait locus for hippocampal volume may exert its effects by regulating the expression of the nearby TESC gene. ${ }^{1}$ This previously not very well studied gene is expressed during development and also moderately during adulthood. Its protein product is involved in cell proliferation and differentiation and thus relevant for hippocampal integrity. ${ }^{2,3}$
In the study reported here, we explored whether this quantitative trait locus also affects hippocampal function, using a previously established neuroimaging paradigm. ${ }^{4}$ As the reliability of our target measure is an important issue in estimating specific genetic effects on hippocampal function, we further performed a test-retest reliability analysis of the identified contrasts to quantify the stability of our paradigm over time. ${ }^{5}$

\section{Methods}

Subjects. A total of 224 German volunteers with grandparents of European ancestry were recruited at Bonn, Mannheim and Berlin and genotyped for rs7294919. The study was approved by the local ethics committee and all participants gave prior written informed consent. No participant reported a lifetime or family history of (neuro)psychiatric disease. Of our sample, 176 participants were rs7294919 TT homozygotes, 42 were CT heterozygotes, and 6 were CC homozygotes. No deviation from Hardy-Weinberg equilibrium was observed $\left(\chi^{2}=0.027, \mathrm{df}=1, P=0.87\right)$. There was no genotype effect on demographic variables, behavioral or psychometric data (Table 1).

\footnotetext{
${ }^{1}$ Department of Psychiatry and Psychotherapy, Charité Universitätsmedizin Berlin, Campus Mitte, Berlin, Germany; ${ }^{2}$ Division of Mind and Brain Research, Charité Universitätsmedizin Berlin, Campus Mitte, Berlin, Germany; ${ }^{3}$ Department of Psychiatry and Psychotherapy, Central Institute of Mental Health, Faculty of Medicine, University of Heidelberg, Mannheim, Germany; ${ }^{4}$ Institute of Neuroscience and Medicine (INM-1), Research Center Juelich, Juelich, Germany; ${ }^{5}$ Department of Biomedicine, University of Aarhus, Aarhus, Denmark; ${ }^{6}$ Department of Genomics, University Hospital Basel, Basel, Switzerland; ${ }^{7}$ Department of Genetic Epidemiology in Psychiatry, Central Institute of Mental Health, Faculty of Medicine Mannheim, University of Heidelberg, Mannheim, Germany; ${ }^{8}$ Institute of Human Genetics, University of Bonn, Bonn, Germany; ${ }^{9}$ Department of Genomics, Life and Brain Center, University of Bonn, Bonn, Germany; ${ }^{10}$ Division of Medical Psychology, University of Bonn, Bonn, Germany and ${ }^{11}$ Department of Psychiatry and Psychotherapy, University of Bonn, Bonn, Germany

Correspondence: Dr S Erk, Division of Mind and Brain Research, Department of Psychiatry and Psychotherapy, Campus Mitte, Charité Universitaetsmedizin Berlin, Chariteplatz 1, Berlin 10117, Germany.

E-mail: susanne.erk@charite.de

${ }^{12}$ These authors contributed equally to this work.

${ }^{13}$ Shared senior authors.

Keywords: ENIGMA; fMRI; hippocampus; imaging genetics; memory

Received 29 May 2013; accepted 31 May 2013
} 
Table 1 Sample characteristics and behavioral measures

\begin{tabular}{|c|c|c|c|c|}
\hline & $T T$ & $C T$ & $C C$ & $\mathrm{P}$ \\
\hline Subjects & 176 & 42 & 6 & \\
\hline $\begin{array}{l}\text { Site } \\
\text { (Bonn/Mannheim/ } \\
\text { Berlin) }\end{array}$ & $79 / 56 / 41$ & $20 / 14 / 8$ & $5 / 0 / 1$ & 0.38 \\
\hline Sex (male/female) & $81 / 95$ & $24 / 18$ & $1 / 5$ & 0.14 \\
\hline $\begin{array}{l}\text { Handedness } \\
\text { (right/left/both) }^{a}\end{array}$ & $159 / 12 / 3$ & $37 / 4 / 0$ & $5 / 1 / 0$ & 0.89 \\
\hline Age (years) & $34.1 \pm 10.0$ & $34.8 \pm 9.9$ & $34.1 \pm 10.0$ & 0.51 \\
\hline $\begin{array}{l}\text { School education } \\
\text { (years) }\end{array}$ & $6.2 \pm 1.0$ & $6.2 \pm 1.3$ & $6.0 \pm 1.1$ & 0.87 \\
\hline Hits recall & $11.6 \pm 1.8$ & $11.4 \pm 1.9$ & $9.8 \pm 1.7$ & 0.07 \\
\hline VLMT early recall & $12.4 \pm 2.4$ & $13.3 \pm 1.7$ & $13.0 \pm 2.5$ & 0.02 \\
\hline VLMT late recall & $12.6 \pm 2.4$ & $13.8 \pm 1.6$ & $13.7 \pm 1.4$ & 0.004 \\
\hline VLMT recognition & $14.4 \pm 1.0$ & $14.6 \pm 0.7$ & $15.0 \pm 0.0$ & 0.19 \\
\hline MWTB & $30.4 \pm 3.5$ & $30.9 \pm 2.8$ & $29.2 \pm 2.5$ & 0.46 \\
\hline BDI & $2.8 \pm 3.3$ & $2.9 \pm 3.1$ & $2.4 \pm 2.9$ & 0.95 \\
\hline STAI-S & $31.3 \pm 0.6$ & $31.6 \pm 1.2$ & $28.5 \pm 2.7$ & 0.95 \\
\hline STAI-T & $35.2 \pm 0.8$ & $35.5 \pm 1.7$ & $36.4 \pm 3.9$ & 0.56 \\
\hline $\begin{array}{l}\text { Hippocampal } \\
\text { volume right }\left(\mathrm{mm}^{3}\right)\end{array}$ & $4535 \pm 473$ & $4615 \pm 454$ & $4441 \pm 414$ & 0.53 \\
\hline $\begin{array}{l}\text { Hippocampal } \\
\text { volume left }\left(\mathrm{mm}^{3}\right)\end{array}$ & $4440 \pm 444$ & $4579 \pm 426$ & $4466 \pm 436$ & 0.19 \\
\hline
\end{tabular}

Abbreviations: BDI, Beck Depression Inventory; C, cytosine; MWTB, Mehrfachwahl-Wortschatz-Intelligenztest; STAI-S, State Trait Anxiety Inventory-state version; STAI-T, State Trait Anxiety Inventory-trait version; $\mathrm{T}$, tyronine (risk allele); VLMT, verbal learning and memory test.

${ }^{a}$ Data missing for three subjects.

Bold values indicate significant $P<0.05$.

DNA extraction and genotyping. EDTA anti-coagulated venous blood samples were collected from all individuals. Lymphocyte DNA was isolated using the Chemagic Magnetic Separation Module I (Chemagen, Baesweiler, Germany). The single-nucleotide polymorphisms rs7294919 were genotyped using a TaqMan $5^{\prime}$ nuclease assay.

Functional imaging task. During functional magnetic resonance imaging (fMRI), subjects completed an episodic recall task that was part of three consecutive memory tasks, that is, encoding, recall and recognition of face-profession pairs. These tasks were part of a functional imaging genetics battery. ${ }^{6}$ The encoding task consisted of 16 face-profession pairs and 24 head contours as control condition with 4 blocks of 4 face-profession pairs and 4 blocks of 6 head contours each. Face-profession pairs were presented for $6 \mathrm{~s}$, and head contours for $4 \mathrm{~s}$. Each block lasted $24 \mathrm{~s}$. Participants had to imagine the person acting in a scene of the written profession and had to indicate whether the profession suited the presented face. During the control condition participants had to indicate which ear of the depicted head contour was larger. The alternating sequence of four face-profession association blocks and four control blocks was presented twice in order to ensure successful encoding. During recall, the task in focus of the present report, faces were presented together with the question whether the depicted person had to complete apprenticeship or academic studies in order to qualify for the respective profession that had been learned during encoding. Subjects had to indicate by button press, which qualification was correct. Stimulus duration and control condition were similar to encoding, blocks were presented only once. For recognition testing faces were depicted together with two written professions and subjects had to indicate which profession was correct. Stimulus duration for recognition was $3 \mathrm{~s}$. The control condition consists of 4 blocks of 4 head contours each (3s). Thus, each recognition block lasted 12s. Similar to recall, blocks were only presented once.

Behavioral and psychopathological measures. On a second day, subjects underwent neuropsychological assessment including testing for verbal intelligence (MWTB, Mehrfachwahl-Wortschatz-Intelligenztest) and memory (VLMT, Verbaler Lern und Merkfaehigkeits-Test, translated verbal version of Rey auditory learning task) and completed versions of the Beck Depression Inventory (BDI) and the State Trait Anxiety Inventory (STAI, Trait version).

Imaging parameters. BOLD $\mathrm{fMRI}$ was performed on a Siemens Trio 3T scanner at the Life and Brain Center, University of Bonn, the Central Institute of Mental Health, Mannheim and the Charité University Hospital Berlin (parameters: 33 slices, axially tilted $\left(-30^{\circ}\right)$, slice thickness $2.4+0.6 \mathrm{~mm}$ gap, FOV $192 \mathrm{~mm}$, TR $1.96 \mathrm{~s}$, TE $30 \mathrm{~ms}$, flip angle $80^{\circ}$ ). High resolution three-dimensional T1-weighted images were acquired with 160 contiguous sagittal slices of $1 \mathrm{~mm}$ thickness (FOV $256 \mathrm{~mm}$, TR $1.57 \mathrm{~s}$, TE $3.42 \mathrm{~ms}$, TI $800 \mathrm{~ms}$, flip angle $15^{\circ}$ ).

Structural image processing. Structural MRI data of this sample were part of the ENIGMA sample. ${ }^{1}$ The volumetric segmentation of the left and right hippocampus was performed with the Freesurfer image analysis suite (version 5.0; http://surfer.nmr.mgh.harvard.edu/). The three mprageimages were fed into the volumetric stream which included motion correction, averaging, removal of non-brain tissue, automated Talairach transformation, intensity nomalization and segmentation of the subcortical white matter and deep gray matter volumetric structures (including the hippocampus) with the help of a probabilistic atlas. ${ }^{7,8}$ Quality control included image inspection, outlier detection via boxplot and subsequent manual editing of the brainmask, details are given in Stein et al. 2012. ${ }^{2}$

Functional image processing. Image processing and statistical analyses were conducted using statistical parametric mapping methods as implemented in SPM8 (http:// www.fil.ion.ucl.ac.uk/spm/software/spm8/). Briefly, images were realigned to a mean image (movement parameters were confined to $<3 \mathrm{~mm}$ translation and $<3^{\circ}$ rotation between volumes), slice time corrected, spatially normalized to a standard stereotactic space (a brain template created by the Montreal Neurological Institute) with volume units (voxels) of $2 \times 2 \times 2 \mathrm{~mm}^{3}$, smoothed with an $8 \mathrm{~mm}$ full-width at half maximum Gaussian filter and ratio normalized to the whole-brain global mean. A first-level fixed-effects model was computed for each participant. Regressors were created from the time course of the two experimental conditions (memory, control) and convolved with a canonical hemodynamic response function. Movement parameters were included in the first-level model as regressors of no interest. For each subject, statistical contrast images of memory versus control were obtained. To test for genetic association, 
these were analyzed using the general linear model in a second-level multiple regression analysis. To test for genetic association, these were analyzed using the general linear model in a second-level multiple regression random effects analysis with age, gender, scanner site and hippocampal volume as nuisance covariates to identify genotype effects on functional activation.

Statistical inference. For all imaging methods, the significance threshold was set to $P<0.05$, corrected for multiple comparisons within our a priori defined anatomical region of interest, the hippocampus. For all analyses, we employed conservative analysis statistics by using family wise error rate, that exert strong control of type I error over multiple comparisons in imaging genetics. Regions of interest were defined a priori using anatomical labels provided by the Wake Forest University PickAtlas (www.fmri.wfubmc.edu/ downloads).

Behavioral and neuropsychological data were analyzed using PASW Statistics 20 (IBM SPSS Statistics, Chicago, IL, USA) and $\chi^{2}$-tests (site, sex, handedness), univariate (age, education, MWTB, performance, BDI) as well as multivariate (VLMT, STAI) ANOVAs with site, age and sex as covariates.

Test-retest reliability of hippocampal activation. In order to quantify the robustness of our activation measures we analyzed test-retest reliability in an independent cohort of young healthy subjects ( $n=20,11$ females, mean age 24.3, mean retest interval 14.5 days) using intraclass correlation coefficients (ICC) as defined by Shrout and Fleiss, ${ }^{9}$ that is, the absolute $(\operatorname{ICC}(2,1))$ and relative $(\operatorname{ICC}(3,1))$ agreement of observations between sessions (for details see Plichta et $a l^{5}$ ). For the purpose of this study, the analysis was spatially restricted to the hippocampal cluster that exhibited significant genotype-dependent activation differences. Outcome measures of interest were ICCs of mean BOLD amplitudes of voxels within this mask. Analyses were performed using PASW Statistics 20 (IBM SPSS Statistics). Consistent with the prior literature, we interpreted ICC values below 0.4 as indicative of poor test-retest reliability, while ICC values between 0.4 and 0.75 were interpreted as 'fair to good' and those above 0.75 as 'excellent', respectively.

\section{Results}

We observed an effect of rs7294919 genotype in the right hippocampus $(x=20, y=-16, z=-16, \quad Z=3.45)$ with activation increasing with the number of $\mathrm{T}$ alleles (Figure 1). Further, in the external VLMT recall test performance decreased significantly with number of $T$ alleles (see Table 1). Rs7294919 had no impact on $\mathrm{fMRI}$ task performance, verbal intelligence, or depression and anxiety measures.

Hippocampal volume did not differ significantly between genotype groups in our (sub)sample. This might be due to the smaller sample size compared with the sample used in the ENIGMA consortium.

The test-retest reliability analysis provided evidence for above average reliability ${ }^{10}$ of our recall task and a very good robustness of the hippocampal activation estimates that
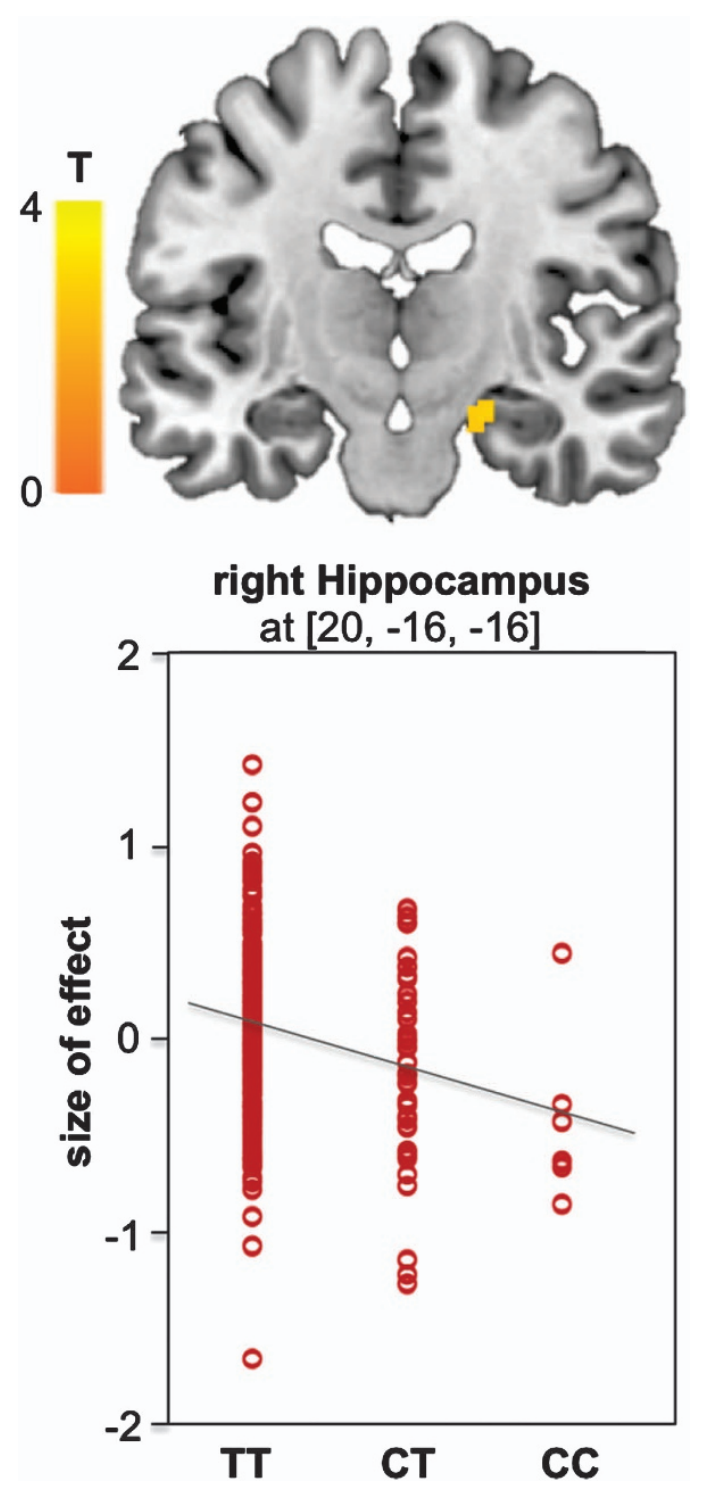

Figure 1 Carriers of the of the risk variant (T) for rs7294919 exhibit significantly increased allele-dosage-dependent activation of the right hippocampus during recall ( $Z=3.45, P<0.05$ family wise error (FWE) corrected for multiple testing across region of interest). Each red dot represents size of effect in one subject.

yielded significant differences in the present study (Intraclass correlations: $\operatorname{ICC}(2,1)=0.73(\mathrm{Cl}: 0.310 .89), \operatorname{ICC}(3,1)=0.73$ (Cl: 0.310 .89$)$ ).

\section{Discussion}

Our findings implicate the variant identified by the ENIGMA consortium at rs7294919 in hippocampal function and episodic memory recall. This underlines the idea that rs7294919 may affect the individual capacity to resist disease in terms of diminishing or boosting hippocampal resources. A test-retest analysis demonstrates a high robustness of our activation estimates in the hippocampus, both in relation to previously established formal criteria $^{9}$ and to the mean reliability of $\mathrm{fMRI}$ studies in general. ${ }^{10}$ 
The observed increase of functional activation during memory recall in carriers of the rs27491929- $T$ risk variant might indicate a compensatory mechanism: as genotype groups did not differ in task performance during fMRI, increased hippocampal activation can be interpreted as overrecruitment needed for similar performance. ${ }^{11}$ Reduced performance in an unrelated and more demanding VLMT might in turn indicate reduced reserve capacity when task difficulty is high. This diminished neuronal reserve could be related to the previously detected association between rs7294919 risk allele and decreased hippocampal volume. ${ }^{2}$

An alternative explanation for increased hippocampal activation could be that hippocampal hyperactivity corresponds to a relative decrease in synaptic inhibition ${ }^{12}$ through neuronal excitotoxicity. ${ }^{13}$ Overactivation of synaptic receptors by excitatory neurotransmitters, for example, glutamate, can lead to high levels of calcium influx into the cell that, if not sufficiently buffered, can activate a number of enzymes leading to structural cell damage. ${ }^{14}$ Interestingly, the protein product of the TESC gene, tescalcin, is a calcium binding protein $^{15}$ and the expression of TESC has been associated with the rs7294919 genotype. ${ }^{2}$ However, the exact nature of the relation between TESC expression and hippocampal volume and function remains to be determined in further translational studies and results of the present study need independent replication.

\section{Conflict of interest}

The authors declare no conflict of interest.

Acknowledgements. Funding for this study was provided by the German Ministry for Education and Research (BMBF) Grant NGFNplus MooDS and by the German Research Foundation (DFG) Grant SFB 636-B7. This work was supported by NIH/NHLBI U01 HL089856, R01 MH087590 and R01 MH081862. M.M.N. also received support from the Alfried Krupp von Bohlen und Halbach-Stiftung.
1. Bis JC, DeCarli C, Smith AV, van der Lijn F, Crivello F, Fornage $M$ et al. Common variants at $12 q 14$ and 12q24 are associated with hippocampal volume. Nat Genet 2012; 44: 545-551.

2. Stein JL, Medland SE, Vasquez AA, Hibar DP, Senstad RE, Winkler AM et al. Identification of common variants associated with human hippocampal and intracranial volumes. Nat Genet 2012; 44: 552-561.

3. Levay K, Slepak VZ. Tescalcin is an essential factor in megakaryocytic differentiation associated with Ets family gene expression. J Clin Invest 2007; 117: 2672-2683.

4. Erk S, Meyer-Lindenberg A, Schnell K, Opitz von Boberfeld C, Esslinger C, Kirsch P et al. Brain function in carriers of a genome-wide supported bipolar disorder variant. Arch General Psychiatry 2010; 67: 803-811.

5. Plichta MM, Schwarz AJ, Grimm O, Morgen K, Mier D, Haddad L et al. Test-retest reliability of evoked BOLD signals from a cognitive-emotive fMRI test battery. Neurolmage. 2012; 60: 1746-1758.

6. Esslinger C, Walter H, Kirsch P, Erk S, Schnell K, Arnold C et al. Neural mechanisms of a genome-wide supported psychosis variant. Science 2009; 324: 605

7. Fischl B, Salat DH, Busa E, Albert M, Dieterich M, Haselgrove $C$ et al. Whole brain segmentation: automated labeling of neuroanatomical structures in the human brain. Neuron 2002; 33: 341-355.

8. Fischl B, Salat DH, van der Kouwe AJ, Makris N, Segonne F, Quinn BT et al. Sequenceindependent segmentation of magnetic resonance images. Neurolmage. 2004; 23 (Suppl 1): S69-S84.

9. Shrout PE, Fleiss JL. Intraclass correlations: uses in assessing rater reliability. Psychol Bull 1979; 86: 420-428.

10. Bennett $\mathrm{CM}$, Miller MB. How reliable are the results from functional magnetic resonance imaging? Ann N Y Acad Sci 2010; 1191: 133-155.

11. Rasch B, Papassotiropoulos A, de Quervain DF. Imaging genetics of cognitive functions: Focus on episodic memory. Neurolmage. 2010; 53: 870-877.

12. Busche MA, Eichhoff G, Adelsberger $\mathrm{H}$, Abramowski D, Wiederhold KH, Haass $\mathrm{C}$ et al. Clusters of hyperactive neurons near amyloid plaques in a mouse model of Alzheimer's disease. Science 2008; 321: 1686-1689.

13. O'Brien JL, O'Keefe KM, LaViolette PS, DeLuca AN, Blacker D, Dickerson BC et al. Longitudinal fMRI in elderly reveals loss of hippocampal activation with clinical decline. Neurology 2010; 74: 1969-1976.

14. Kandel ER, Schwartz JH, Jessell TM, Siegelbaum SA, Hudspeth AJ. Principles of Neural Science. 5th edn McGraw-Hill Medical: New York, NY, USA, 2013.

15. Gutierrez-Ford C, Levay K, Gomes AV, Perera EM, Som T, Kim YM et al. Characterization of tescalcin, a novel EF-hand protein with a single $\mathrm{Ca} 2+$-binding site: metal-binding properties, localization in tissues and cells, and effect on calcineurin. Biochemistry. 2003; 42: 14553-14565.

Translational Psychiatry is an open-access journal published by Nature Publishing Group. This work is licensed under a Creative Commons Attribution-NonCommercialNoDerivs 3.0 Unported License. To view a copy of this license, visit http://creativecommons.org/licenses/by-nc-nd/3.0/ 